\title{
Designing Pervasive Interactions for Ambient Guidance with Situated Flows
}

\author{
Fahim Kawsar, Gerd Kortuem, Bashar Altakrouri \\ Computing, Lancaster University, UK \\ \{kawsar,kortuem,b.altakrouri\}@comp.lancs.ac.uk
}

\begin{abstract}
Effectively guiding people in complex and highly dynamic work environment requires advances in high-level declarative activity models that can describe the flow of human work activities and their intended outcomes, as well as novel user interface models for distributing guidance information across time and space. This paper describes a new line of research aimed at developing a new programming and human interface approach for pervasive systems based on high-level models of human activities, socalled situated flows, and mobile projector interfaces for uncovering task information embedded in physical environments.
\end{abstract}

\section{Introduction}

Over the past years research in pervasive computing has demonstrated the potential of context-aware and proactive technologies for improving human work performance. Examples include systems for tracking and automatically recording task performance in industrial maintenance scenarios [7,9], measuring and informing workers about their exposure to equipment vibrations [6] and context-aware information capture and presentation at hospitals $[1,5]$. Pervasive work support systems make use of sensors and handheld and wearable devices to analyze work activities in real-time and to provide users with relevant and timely information pertaining to their work. Projecting in the future we can imagine that future work environments will be densely instrumented and be able to understand minute details of work activities and processes.

Yet while there have been great advances in sensing, great challenges for the development of pervasive work support systems remain: the first challenge relates to the lack of technology-independent and transferable models of human work activities. Activity recognition approaches are driven from the bottom up and use models that are highly dependent on algorithms and technologies; they are not suitable as declarative modeling tool for specifying organizational work processes. Workflow technologies based on BPEL [8] and other languages provide an interesting starting point for the development of declarative activity models, yet existing approaches lack features to express physical context (location etc) and are not suited for integration with activity recognition technologies.

The second challenge relates to the design of distributed and embedded interaction techniques and user interfaces to effectively support people in demanding work environments such as hospitals and industrial plants. While there has been much progress on mobile and wearable device interfaces, the question of how to distribute information in a physical environment across time and space considering user context and work processes with the goal to maximize human performance has not yet been tackled. Yet, pervasive interaction mechanism, i.e., strategies for determining when, where and how to present work-related information in a situated fashion and corresponding interaction techniques is at the core of future pervasive work support systems.

In this paper we report our initial experience on a new line of research aimed at developing a new programming and human interface approach for pervasive systems based on high-level models of human activities, so-called situated flows. Situated flows model human work processes as a set of physical actions glued together by a plan (a set of transitions), which defines how activities should or could be performed to achieve a specific outcome. In contrast to traditional workflows, situated flows are situated and context-aware: they are linked to physical entities like equipment and people, moving with them through different environments, thereby reacting to and being influenced by their context. We use situated flows to drive the distribution, presentation, and interaction of workspace task related information in pervasive work environments. We termed such situated assistances as "Ambient Guidance" that is driven by well defined strategies and situated flows. In our first prototype body-worn context-aware projectors allow people to uncover flows and task information embedded in the surrounding physical environment. This provides for a seamless user experience where task information is virtually overlaid on physical entities it relates to and aids people to achieve their goal in structured workplaces.

This paper focuses on user interfaces and ambient guidance strategies. We first describe situated flows and its implications in designing interaction. This is followed by the description of the ambient guidance system that is driven by situated flows. We conclude the paper by reporting our initial insights and discussing the future avenue of our research.

\section{Situated Flows}

A situated flow (flow, for short) is a high-level programming language for modeling real-life processes and human activities. It consists of a set of actions glued together by a plan (a set of transitions), which defines how actions should be performed to achieve some goal under a set of constraints $[10,11]$. 
Actions model physical activities of people or digital processes. For example, for a nursing home scenario we identified situated flows that model the daily rounds of a nurse in a hospital, the daily care schedule of a patient and the operation procedures or handling instructions of various pieces of medical equipment (Figure 1).

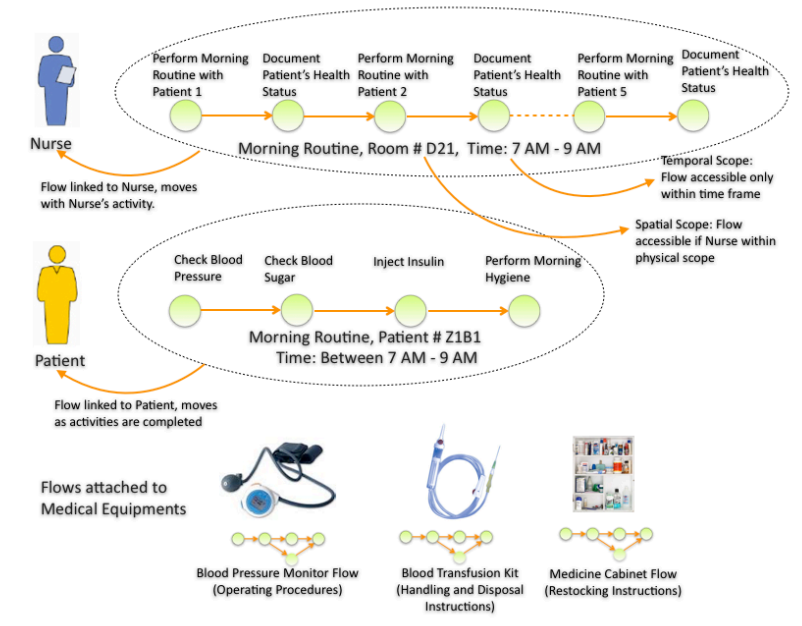

Figure 1. Situated flows in Nursing Home Scenario. Patient flow, nurse flow and equipment flows.

Situated flows are closely related to classical workflows [8], yet they differ in that they are situated and contextaware: they are (logically or physically) linked to real-world entities like people and objects, being carried by them or moving with them through physical environments. Flows are executed in parallel to the real-world actions they describe: when an action that is described in a flow is performed in the real world (by a person), the flow progresses one step.

From a human point of view, situated flows define opportunities for action. A flow embedded in an object or room defines tasks and actions that can or should be performed with the object or in the room. An action can be a physical action such as dispensing medication or a digital action such as turning on a machine.

\subsection{Situated Flow Driven Interaction}

Classical human computer interaction is framed against a dynamic transfer function that couples input and output through a feedback loop. More recently as a natural consequence of engraving computation in the fabrics of environment, this input space is enhanced with richer modalities like situational contexts of humans (e.g., location, activity, etc.) to modulate system outputs, and to improve the dynamics of interaction by providing services proactively. Context driven systems usually consider temporal contexts, and in some cases (for systems with learning capabilities) historical contexts to model the interaction. However these systems usually do not predict the upcoming aspects of context beyond current interaction point. As a consequence, they are not adequate in providing effective guideline, and adapting interfaces over time by looking probable user activities and associated interaction points ahead of the execution. In addition context driven systems are specifically framed for supporting a particular activity. However, human activities are often interleaved, and very much ad-hoc even in a controlled environment. Thus, rule based systems driven by contexts provide limited opportunities to maintain the interface consistency for supporting such interleaved interactions. Situated flows as described above can contribute to address these issues. Essentially a situated flow represents a collection of sequential actions stitched together with a plan, where each action is interdependent and might offer one or more interaction opportunities. Thus it enables an action and it's associated interaction points to be analyzed, modeled, and adapted ahead of the execution. Specifically, interaction driven by situated flows brings three optimizations, these are:

- A situated flow enables a system to provide intelligible feedback by considering not only the present and historical context, but also future planning. This essentially enables a system to guide its user to perform the activities according to the flow. For prescriptive system, a flow driven interaction can thus provide appropriate feedback to user when his/her activities deviate from the actual plan and can guide the user. However for a descriptive system where activities are typically modeled in an ad-hoc fashion, a flow may offer support in a more opportunistic fashion.

- A situated flow models a collection of activities that are performed over time. However, due to the awareness of the entire activity space, a flow can optimize the upcoming interaction points (both immediate and later) based on the current and previous interaction patterns. This means, it provides an interface designer with the flexibility to adapt future interface references.

- A situated flow is particularly useful to maintain the consistency of the interfaces of interaction points. Usually, human activities are interleaved, and two consecutive human tasks may be part of two different activities with different goals. Having a flow associated with an interface, allows designers to ensure that the consistency of the interface is maintained over time, when human actions are switched from one activity to another.

\subsection{Combination of Flow Driven Interaction}

In the previous section, we have described three different kinds of situated flows associated in a pervasive workspace environment. The organization flow represents the multiplexed version of personal and object flows. One way to characterize the interactions driven by these flows is by looking at ways in which these flows coupled together to 
interact in a pervasive computing setup. In the following we describe four different interaction use cases combining these flows.

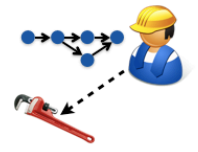

(a)

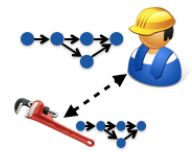

(c)

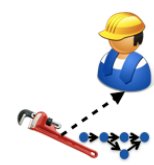

(b)

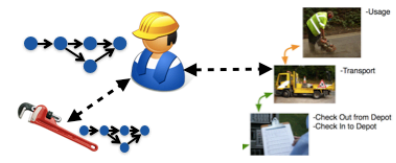

(d)
Figure 2: Combination of Flow Driven Interaction

- $\quad$ Figure 2(a) depicts the first case primarily driven by a personal flow. A person carries one or multiple flows that describe the sequence of activities that the person needs to perform to achieve the goals. When a person reaches a point in its flow where he/she needs to interact with an object, personal flow can interact with the respective objects to create a dynamic coupling enabling the object to support the activities within the flow to advance to the next course of actions towards achieving the objective.

- Figure 2(b) highlights a variant of case 1, where physical objects are populated with one or multiple objects' flows that can support personal flows or organizational flows. However, in this case, a person do not possess any flow, but at the point of interaction (i.e., when the object is used by the person) the object flow is associated with the person and according to the activities performed by the person, the object flow is advanced to the next course of its state.

- Figure 2(c) depicts the conjugal of the above two use cases, a person may carry one or multiple flows, and at a certain point of his/her activities he/she might reach a situation where he/she needs to interact with one or multiple objects populated with one or multiple object flows. In this case, two flows are merged; ideally, the object flow becomes a sub-flow of the personal flow. However, in the case when the object flow represents a part of a larger organizational flow, the personal flow coupled with the object flow can become the sub-flow of the larger organizational flow.

- The final case is the auxiliary flow driven by the coupling between personal and object flows as shown in figure 2(d). In this case, the auxiliary flow depends on the interaction between the personal and object flow and based on the transition of their interaction, the auxiliary flow is advanced to next stage to meet its objective.

Assuming an organization has defined flows and linked them to entities, people and locations (thereby creating a physically dispersed business process model), the key question we investigate is: How can a person discover flows in the surrounding physical environment and act upon them? Alternatively phrased: How can situated flows be used to effectively guide peoples' actions and help them achieve work-related goals?

In the next section we present an ambient guidance system that address this question.

\section{Ambient Guidance System}

Our Ambient guidance systems consist of two components: guidance strategies for deciding which information should be accessible and when, where and how it should be presented in the environment. This is used to uncover task information embedded in physical environments and present the situated guidance information using a mobile-projected interface.

\subsection{Ambient Guidance Strategies}

Flows represent context-specific prescriptions for how activities and tasks are supposed to be done or how equipment is to be operated. Mobile projection interfaces make it possible to expose activity and task information to users. Yet in order to effectively guide people, i.e. help them achieve goals defined by flows, it is not enough to simply present people with every single task they come across. Effective guidance requires a guidance strategy that defines:

- Which tasks and activities are exposed to people (by selecting flows and activities from within the local scope of the user)

- When and where guidance information is presented.

- How to visually present guidance information.

- How to cope with situations in which people do not follow flow descriptions.

In order to cope with disparate requirements of application domains we developed two levels of generic guidance strategies.

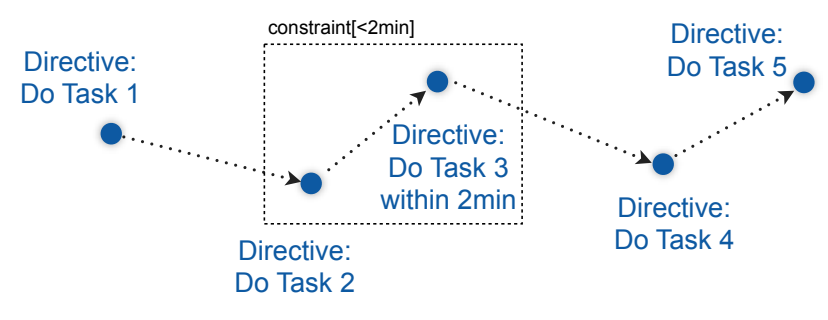

Expected behaviour (specified by flow)

Figure 3. Directive Guidance

\subsubsection{Directive Guidance}

Directive guidance (Figure 3) is a strategy that presents users with just-in-time notifications (directives) of the next activities to be done (as defined by the flow). To be precise, directives are generated and presented to users just before an activity has to be performed. For example, in a hospital 
scenario, before and during preparing medicines for a patient, it is beneficial to provide an updated (if any) prescription guide to the nurse. This could be useful for certain circumstances, e.g., immediately after the nursing shift change.

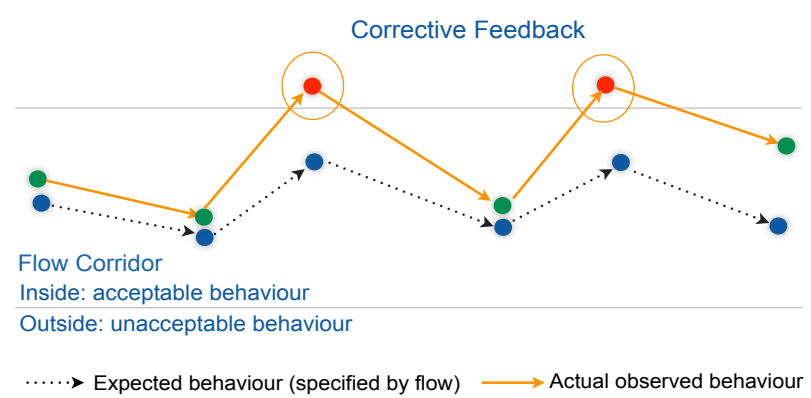

Figure 4. Corrective Guidance

\subsubsection{Corrective Guidance}

Corrective guidance (Figure 4) is a strategy that assumes that people have satisfactory understanding of what they have to do and that they don't require constant reminders. Instead this strategy only presents users with guidance information when the flow system detects significant deviations from the plan. This is visualized in Figure 4: a flow corridor defines how much an actual activity may deviate from the one prescribed by the flow. If an activity falls outside the flow corridor, the flow system issues corrective feedback to inform users of the deviation and motivate the user to follow the plan as described. For example, in a hospital scenario, if a nurse accidently overlooks or delays a scheduled checkup or medication of a patient, the guidance system kicks in with a reminder. In an industrial scenario, if a worker performs critical operations in the wrong order and thus incurs a safety risk, the guidance strategy determines appropriate counter measures (for example undoing of already taken steps) and decides how to inform the worker. The corrective plans can be dynamically generated from the flow model and current flow state.

Directive guidance is a suitable strategy in training scenarios or when work activities must be performed exactly as described (for example for safety reasons). Corrective guidance, on the other hand, is more suited for expert users and relaxed application domains, where deviations are to be expected or can be tolerated.

\subsection{Projected Interfaces for Ambient Guidance}

Designing user interfaces for ambient guidance systems raises considerable design challenges due to i) peoples' primary engagement in physical activities, ii) mobility support, and iii) temporal and spatial value of information. Thus traditional handheld interfaces and emerging distributed public displays are not well suited. Conversely, pervasive computing has instigated a transformation of our environment into an ecological synergy of networked smart objects $[2,4,13]$. This enables us to distribute and embed flows into physical workplace objects and to utilize them as a natural interaction points for ambient feedback and guidance. The recent progression of mobile projectors and projector phones [3] provide an effective solution for realizing object and environmental interfaces. Fundamentally, mobile projection technology overcomes the output limitation of flow-embedded objects by turning them into dynamic information displays.

Accordingly in our ambient guidance system for the dynamic complex workplace environment, we have adopted the following design decisions:

1. Flow Embedded Smart Object and Space: Physical workplace and smart objects are embedded with flows. Users interaction with the object and/or environment triggers execution of flows and stimulates the information presentation for guiding users.

2. Wearable Projector Augmented Mobile Phones: Users wear a projector phone that converse with the surrounding environment and triggers information display projection contextually on the appropriate surface and objects considering the activity in context.
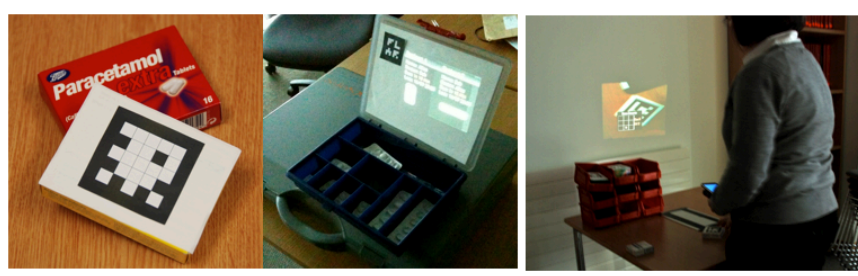

(a) Smart Medicine Box and Medicine Tray. (b) Guidance Information Projected on the Surface

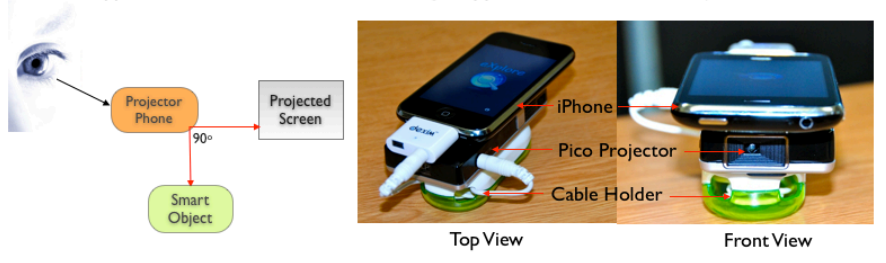

(c) Pico Projector Augmented Mobile Phone drives the presentation and interaction

Figure 5. Ambient Guidance System

The combination of these provides a seamless ambient guidance system as users can focus on their primary activity, and can switch their attention to the information display contextually when needed, users can be mobile and perform organizational activities both indoor and outdoor and finally users are offered spatially and temporally appropriate guidance in an unobtrusive fashion.

Our current implementation uses 2D bar codes to identify objects and projection surfaces. Figure 6 shows the current prototype of our system, where a flow-enabled medicine tray (Figure 5 (a)) and workplace surface present contextual ambient guidance to the user (Figure 5 (b)) and Figure 5(c) shows the interaction mechanism and hardware setup utilized for the interaction. 


\section{Discussion}

In the previous sections, we have presented the primary building blocks for facilitating ambient guidance in workplace environment. Situated flow accommodates the structural foundation for instrumented smart objects to actively guide people leveraging a projected user interface multiplexed by context-aware guiding strategies. Currently, we are exploring the application of such guidance system in a hospital to support the daily activities of nurses. As mentioned in the earlier sections, situated flows distributed across the instrumented environment model the activities of the nurses, and a fine-grained activity recognition system [7] works atop these flows to provide accurate contexts that trigger the guidance. In the current prototype nurses are assumed to carry a projector camera phone that act as the primary interaction channel for the system, i.e., depending on the context of the nurse (e.g., state of the flow, current activity, location, position, etc.) information notification is projected. Our early insights with the guidance system open up a range of interesting research issues that need thorough investigations to formulate a concrete understanding on the impact of such ambient guidance system in critical workplaces. Some of these issues inline with our future work are discussed below.

Discovery and Association of Flow: The fragmentation and distribution of situated flows across time and space in multitude of instrumented smart objects put forth a range of issues related to ambient guidance. For instance, at this moment it is not clear what defines the scope of a flow and how this scope contributes to the discovery and association of flows to smart objects and people, i.e., how to selectively present a set of tasks and associated guidance to users? What context parameters determine the granularity of this exposition of activity? What degree of autonomy is optimum? Our immediate future avenue of work includes a deeper investigation of these issues from a user interface perspective, and to gain insights on the influence of situated flow discovery and association mechanisms in interface design.

Spatio-Temporal Information Distribution: Mobile projected interface is an exciting emerging technology that has potential to bring substantial advancement in building pervasive interfaces distributed across physical spaces. However, there are several design and usability issues that need to be addressed to fully reap the benefits of this technology. Our early experiences with the mobile projected interfaces in guiding people exposed some of these issues. It is essential to have a formidable understanding on where to project information, when and for how long? For example, guidance notification could be projected on the nearest flat surface, or on the floor, or on the object that the user interacting with. It is not clear what parameters contribute in determining the target projection area and what degree of control users should have to overwrite system's decisions. Currently, we are exploring these design questions to frame design guidelines for projected interface in workplace environment.

\section{Conclusion}

This paper discussed our initial experiences with a flow driven ambient guidance system for a demanding workplace environment. We presented the fundamental concepts and an early stage prototype and identified key research issues as an agenda for future research.

Acknowledgment: The European Commission has funded this research. (EC 213339 ALLOW).

\section{References}

[1] Bardram, J., Hansen, T., Mogensen, M., and Soegaard, M. Experiences from Real-World Deployment of Context-Aware Technologies in a Hospital Environment. Proceedings of Ubicomp 2006. pp 369-386.

[2] Gellersen, H. W., Schmidt, A., and Beigl, M. Adding some smartness to devices and everyday things. In 3rd IEEE Workshop on Mobile Computing Systems and Applications (WMCSA 2000), pages 3-10.

[3] Hang, A., Rukzio, E., Greaves, A. Projector phone: a study of using mobile phones with integrated projector for interaction with maps. Proceeedings Mobile HCI 2008, pages 207-216.

[4] Kawsar, F., Fujinami, K. and Nakajima, T. Augmenting everyday life with sentient artefacts. 2005 joint conference on Smart objects and ambient intelligence: innovative contextaware services: usages and technologies (sOc-EUSAI 2005), pages 141-146.

[5] Kjeldskov, J. and Skov, M. B. 2007. Exploring contextawareness for ubiquitous computing in the healthcare domain. Personal Ubiquitous Comput. 11, 7 (Oct. 2007), 549-562

[6] Kortuem G., Ball, L., Busby, J., Davies, N., Efstratiou, C. Iszatt-White, M., Finney, J., Kinder, K., Sensor Networks or Smart Artifacts? An Exploration of Organizational Issues of An Industrial Health and Safety Monitoring System. Proceedings of Ubicomp 2007, Innsbruck, Austria, 2007.

[7] Kunze K., Wagner F., Kartal E., Kluge E. M., and Lukowicz P., Does Context Matter? - A Quantitative Evaluation in a RealWorld Maintenance Scenario. Conference on Pervasive Computing, 2009, May 11-14, Nara, Japan.

[8] Leymann, Frank; Roller, Dieter: Modeling business processes with BPEL4WS. In: Information Systems and e-Business Management (ISeB). Vol. 4(3), Springer, 2006.

[9] Stiefmeier, T., Roggen, D., Ogris, G., Lukowicz, P., Tröster, G. Wearable Activity Tracking in Car Manufacturing. IEEE Pervasive Computing. Vol. 7:2, 2008, 42-50.

[10] Bucchiarone, A., Lafuente, A., Marconi A., and Pistor, M. A formalisation of Adaptable Pervasive Flows. 6th International Workshop on Web Services and Formal Methods (WSFM'09), 2009.

[11] Marconi, A., Pistore, A., Sirbu, A., Eberle, H., Leymann, F. and Unger, T. Enabling Adaptation of Pervasive Flows: Builtin Contextual Adaptation. Joint ICSOC\&ServiceWave 2009 Conference, The 7 th International Joint Conference on Service Oriented Computing (ICSOC 2009).

[12] Wolf, H., Herrmann, K., and Rothermel, K. Modeling Dynamic Context Awareness for Situated Workflows. Proceedings OTM 2009 Workshops, Springer LNCS 5872.

[13] Kortuem, G., Kawsar, F., Sundramoorthy, V., Fitton, D. Smart Objects as Building Blocks for the Internet of Things. IEEE Internet Computing, January/February 2010 (vol. 14 no. 1), pp. 44-51. 